\title{
The practical application of subwavelength focusing elements in the EHF imaging system
}

\author{
Sergey Zhakupov ${ }^{1}$, Alexander Badin ${ }^{1, *}$, and Alexander Berdyugin ${ }^{1}$ \\ ${ }^{1}$ National Research Tomsk State University, Radiophysics Department, 634050 Tomsk, Russia
}

\begin{abstract}
This paper presents the results of modeling the processes of interaction of an electromagnetic wave of the EHF range with focusing elements made of materials with a refractive index $n$ in the range from 1.2 to 2 . Based on the obtained simulation results using additive technologies, an equilateral parallelepiped of acrylonitrile butadiene styrene with a refractive index was created $n=1.62$. After which it was added to the EHF imaging system, and its focusing properties were analyzed. As a result of the experiments, pictures were obtained of the dependence of the intensity of electromagnetic radiation on the surface of test samples on the presence of a given focusing element in the system.
\end{abstract}

\section{Introduction}

Since the end of the twentieth century, the technology of extremely high (EHF) and hyperhigh frequencies has been actively developing. Owing to the special properties of EHF radiation (in particular the terahertz range), it has found its application in such areas of human activity as: safety systems, medicine (diagnostics of the upper layers of human skin), spectroscopy and flaw detection (both industrial, for non-invasive analysis of production products, and private, to visualize the structural component of objects) [1-5]. However, modern imaging methods have their drawbacks, primarily associated with low resolution. Therefore, it is of great interest to create new focusing elements that can achieve a result close to the diffraction limit and even overcome it.

In this paper, we present the results of modeling the process of interaction of electromagnetic radiation with a cubic subwavelength element, as well as practical application in a terahertz image system based on a BWO spectrometer.

\section{Objects of research}

One of the promising areas of analysis of heterogeneous materials is "terahertz imaging". It is a visualization of the internal structure of the object. In the particular case, when using a terahertz spectrometer with a source of continuous radiation, the intensity distribution and phase shift of the electromagnetic radiation passing through the sample are analyzed. This method allows you to clearly demonstrate all the internal heterogeneities of the investigated object, starting from hidden defects - cavities, cracks, ending with

\footnotetext{
*Corresponding author: thzlab@mail.ru
} 
the identification of the material composition, thus allowing a detailed contactless analysis of heterogeneous objects. One of the qualitative indicators of this method is its spatial resolution. This characteristic can be interpreted as the minimum linear or angular distance between two points at which they do not merge into a single unit and the detector is able to distinguish them.

When using a terahertz spectrometer, the spatial resolution can be increased in several ways. In the first case, the radiation frequency can be increased, but this will lead to an increase in the absorption of energy of the electromagnetic wave in the sample. This inevitably leads to a decrease in the total radiation intensity, and the detector will not register a useful signal. The second method is constructive in nature, and it consists in improving the focusing system - minimizing the spot diameter of a focused quasi-optical beam. The second option is the most interesting.

At present, several structures are known that make it possible to create so-called photonic jets that have sub-wave sizes of the scattering spot and, in some cases, are close and even overcome the diffraction limit. One of these structures is a dielectric microsphere, which, depending on the diameter and composite material, i.e. the refractive index allows the formation of photonic jets of the order of half the wavelength [6,7]. Also interesting is the fact of using spheres with a linear gradient change in the refractive index [8], which makes it possible to establish the focusing depth. However, for the most part, the information presented on the interaction of microspheres with electromagnetic radiation is the result of computer simulation. In this connection, the question arises about the placement of these microspheres in the $\mathrm{THz}$ spectrometer system and the degree of influence of the elements in contact with them.

Another conical structure used for focusing terahertz radiation is a cone-shaped metal conductor of a millimeter length [9]. Capturing the electromagnetic waves incident on them, these conductors act as antennas and transform them into limited fields in the near zone at the conical apex. When this field is changed by an inhomogeneous object, the scattered radiation carries information about the local dielectric properties of the sample. This information is recorded by a remotely mounted detector and can be converted into an image. The presented $\mathrm{THz}$ imaging method demonstrates a record resolution $(\sim 40 \mathrm{~nm})$, while achieving high image contrast and the speed of signal capture and processing.

Continuing the theme of focusing electromagnetic radiation, it is also worth mentioning the presence of so-called metamaterials. The assumption is known that, using metamaterials with a negative refractive index, the diffraction limit can be overcome due to the properties of the frequency dispersion of the "left" medium. An example of the use of a metamaterial can be a spectral-broadband lens with a gradient change in the refractive index [10], which allows focusing the $\mathrm{THz}$ radiation with a spot of dispersion equal to the wavelength. It is worth noting that these lenses have a subwavelength thickness and high focusing power, which allows the creation of compact $\mathrm{THz}$ imaging systems with high spatial resolution. The structure of the metamaterial is an alternation of layers of benzocyclobutene and structured copper. The copper sheet contained an array of rings (slots), and the gradient transition of the refractive index was carried out due to a smooth change in the radius of the rings from the center of the sheet to its borders. Although the focusing of radiation to a subwave level was achieved in this work, in comparison with microspheres and microsteps, amplification of radiation intensity at the output of the focusing structure does not occur.

The last structure under consideration capable of focusing electromagnetic radiation is a dielectric cube [11]. Using these structures, it is possible to achieve the formation of a photon stream in the near zone of the cube with a full width at half amplitude (FWHM) 
in the range from 0.4 to 0.7 and an increase in the intensity of incident radiation up to 15 times. This structure does not have the property of broadband since in order to achieve the desired result it is necessary that the edge of the cube be approximately equal to the wavelength of electromagnetic radiation incident on it. However, we will give preference to this element in our experiment, because it has the most promising properties, and is quite simple in physical execution.

\section{Simulation results}

To select the material from which the focusing element will be made, four simulations of the process of interaction of an electromagnetic wave with a cube with a different refractive index in the range from 1.2 to 2 were carried out (Fig. 1).

For simulation, a frequency of $42 \mathrm{GHz}$ was chosen. The choice of this frequency is due to the use of a backward wave lamp in a practical experiment, as well as the desire to use a cube of maximum dimensions in the future, because it's easiest to work with.

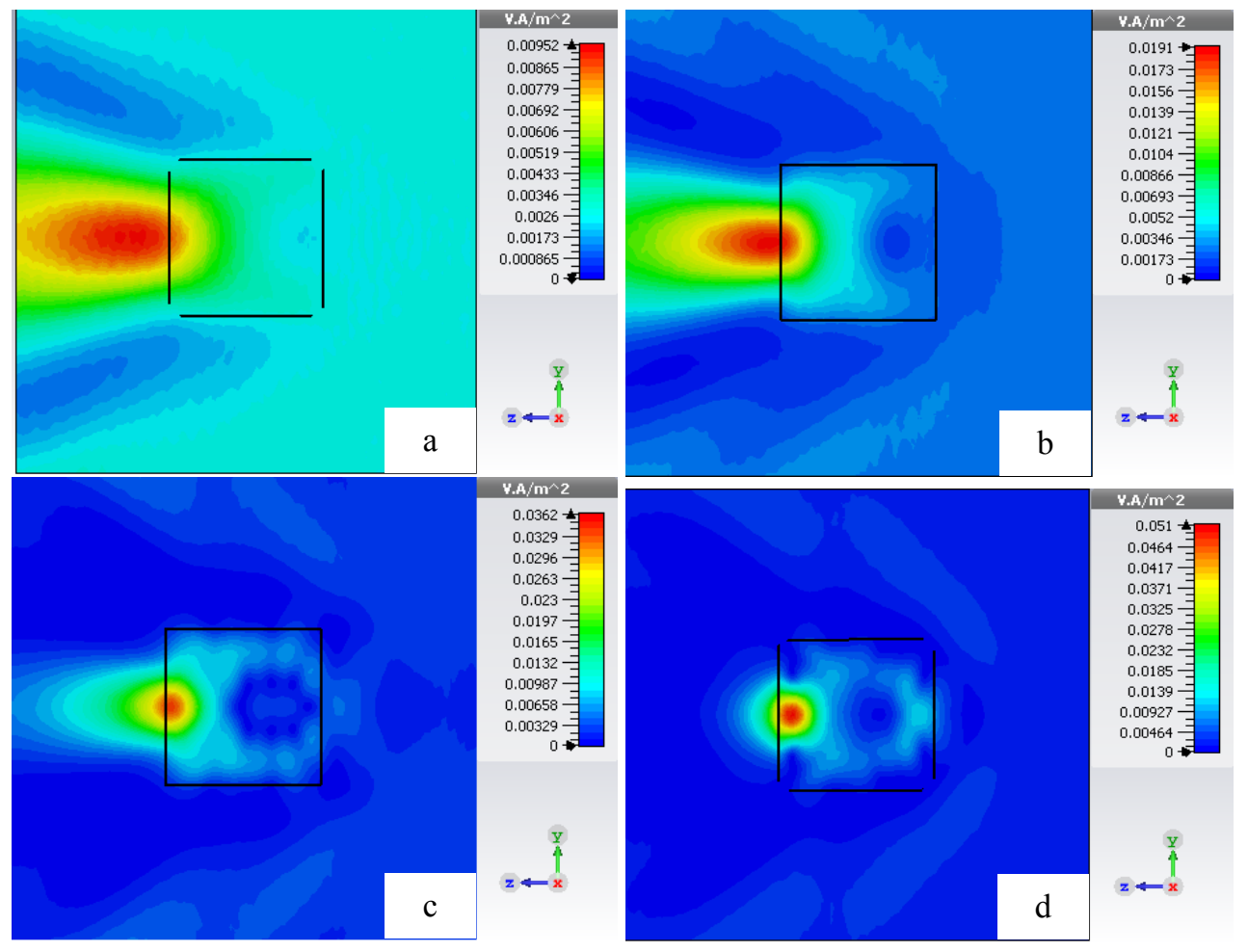

Fig. 1. The distribution of the electromagnetic field in the near zone of the dielectric cube at a frequency of 42 GHz: A) $n=1.2$; B) $n=1.41$;C) $n=1.73$; D) $n=2.00$.

It can be seen from the simulation results that, with an increase in the refractive index, the scattering beam narrows and the intensity peak first shifts to the surface of the cube, and when $\mathrm{n}=2$ it appears inside it. In addition to bias, an increase in the refractive index leads to an increase in the maximum intensity of the formed beam. Thus, the most favorable option is to use cubes with a refractive index in the range from 1.4 to 1.7 , because in this range, the formation of the "photon stream" optimal in width occurs, and the peak of intensity will also be outside the cube. 


\section{Experiment results}

Taking into account the requirements for the cube, it was decided to use the additive technology method (3D layer-by-layer printer) using ABS as a raw material (acrylonitrile butadiene styrene) plastic. ABS plastic has a refractive index of $n=1.62$, and is excellent for creating such structures due to ease of use and subsequent machining. Taking into account the values of the used frequency, the cube was created with dimensions of $7 \times 7 \times 7$ $\mathrm{mm}$. In the terahertz imaging system, it was fixed in a two-dimensional positioning module mounted on the basis of a standard sample retainer (Fig. 2).
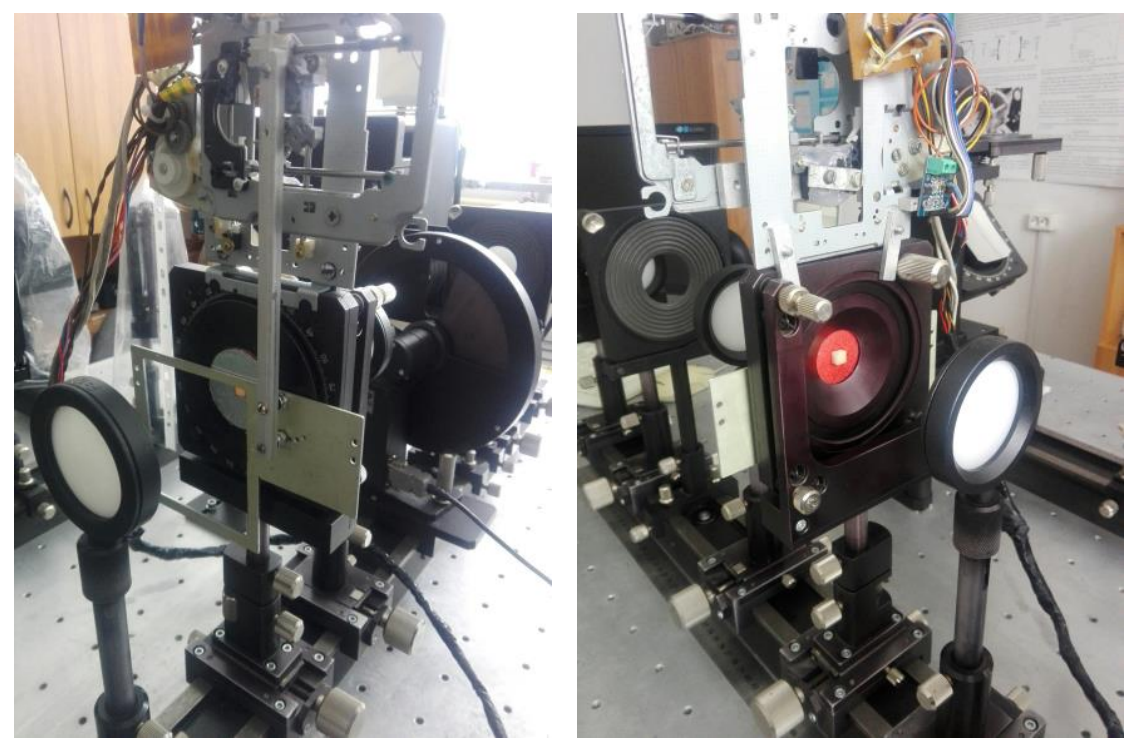

Fig. 2. The location of the focusing element in the terahertz imaging system.

To demonstrate the resolution, a test sample was made, consisting of a sheet of paper 100 microns thick with an array of rhombus shapes made of aluminum foil on top (10 microns thick). The array consisted of three identical vertical rows, each of which consisted of three figures measuring (from bottom to top) $7 \times 4 \mathrm{~mm}, 9 \times 5 \mathrm{~mm}$ and $14 \mathrm{x} 7 \mathrm{~mm}$ (Fig. 3a).

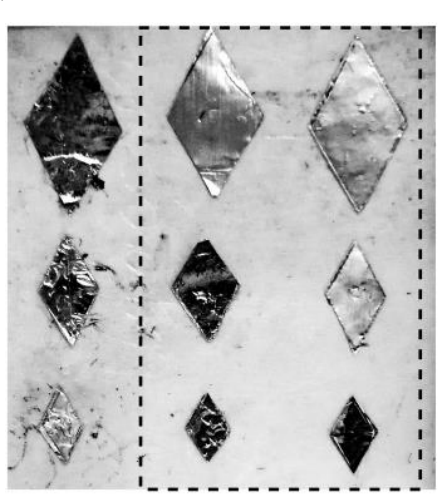

a

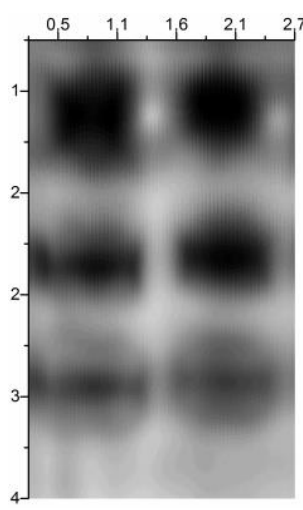

b

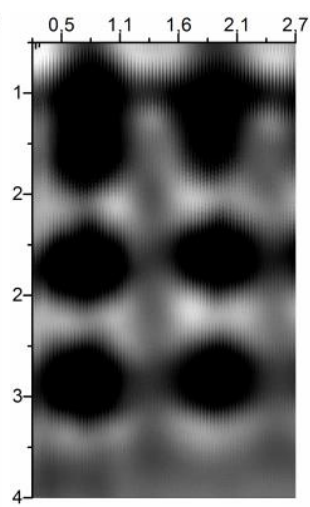

c

Fig. 3. Appearance of the test sample (a); image of a scanned sample without (b) and with a focusing element (c) at a frequency of $42 \mathrm{GHz}$. Black color corresponds to the minimum signal detector, white to the maximum. 
The sample was also fixed in a two-dimensional positioning system so that it moved along the expected peak of the intensity of the beam formed by the cube.

As a result of the experiment, two patterns of the intensity distribution along the surface of the sample were obtained with (Fig. 3b) and without using a cube (Fig. 3c). As can be seen from the results of the experiment when using the cube, we got a more contrast image, we can confidently determine the presence and position of geometric shapes, which is not possible without the use of an additional focusing element. However, the shape of the rhombuses remains distorted, which may be due to undesirable reflection of the electromagnetic wave along the boundary of the test figures.

For the second experiment, a simpler sample was made consisting of metal strips (aluminum foil with a thickness of $10 \mu \mathrm{m}$ ) spaced apart by a width greater than the wavelength Figure 4.
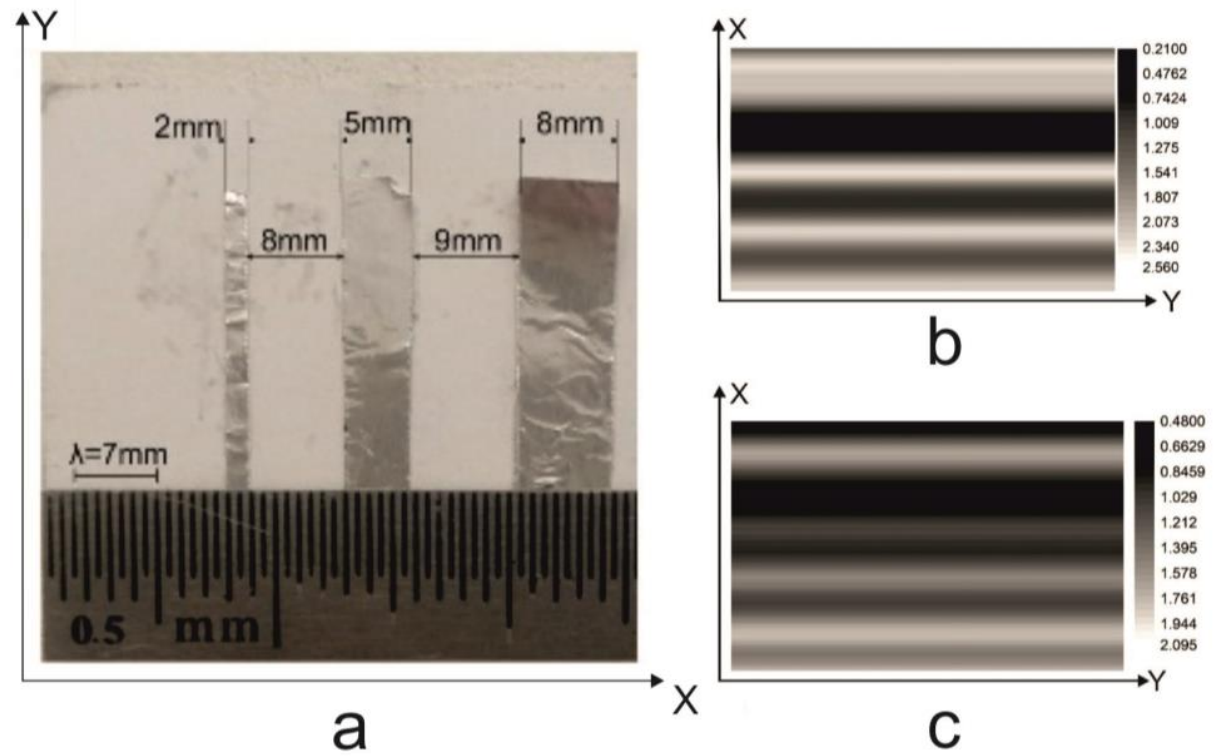

Fig. 4. Image of a test sample with metal stripes (a); The result of visualization of the intensity distribution matrix of transmitted electromagnetic radiation in a two-dimensional plane with (b) and without using a focusing element (c). Shades of gray characterize the signal level from the detector in absolute units.

From the results of the experiment it is seen that when using the cube, we again get a fairly contrasting image of the object. In addition, it is clear that without the use of a cube, imaginary (false) bands appear due to diffraction and re-reflection of the electromagnetic wave. And when using the cube, we can even find that band whose width is 3.5 times less than the wavelength.

Thus, as a result of experiments, the effectiveness of using a subwave cubic element in a terahertz image system is clearly confirmed.

\section{Conclusion}

The report experimentally shows the possibility of increasing the resolution of modern systems of radio-wave imaging of materials through the use of additional focusing elements, in particular a sub-wave cubic object. The results of this work can be applied to improve non-contact inspection systems where continuous radiation sources are used. 


\section{References}

1. Federici J. F., Schulkin B., Huang F., Gary D., Barat R., Oliveira F., Zimdars D., Semicond. Sci. Technol. E 20, 7 (2005)

2. Zhakupov S.N., Badin A.V., Publisher "Proceedings of APEIE - Actual Problems Of Electronic Instrument Engineering". E 1, 6 (2018)

3. Guillet J. P., Wang K., Roux M., F. Fauquet, F., Int. Conf. Infrared, Millimeter, Terahertz Waves, IRMMW-THz. E 7, 2 (2016)

4. Nguyen Pham H. H., Hisatake S., Minin O. V., Nagatsuma T., Minin I. V., APL Photonics. E 2, 5 (2017)

5. Chernomyrdin N. V., Schadko A. O., Lebedev S. P., Tolstoguzov V. L., Kurlov V. N., Reshetov I. V., Spektor I. E., Skorobogatiy M., Yurchenko S. O., Zaytsev K. I., Appl. Phys. E 110, 22 (2017)

6. Lu Y.F., Zhang L., Song W. D., Zheng Y. W.\& Luk'yanchuk B. S., JETP Letters. E 72, 9 (2000)

7. Yang Y., Liu H., Yang M., Cui B., Zhang W., Appl. Phys. Lett. E 113, 3 (2018)

8. Kong S.-C.,Taflove A.\& Backman V., Optics Express. E 17, 5 (2009)

9. Huber A. J., Keilmann F., Wittborn J., Aizpuru, J.\& Hillenbrand R., Nano Letters. E 8, 11 (2008)

10. Neu J., Krolla B., Paul O., Reinhard B., Beigang R.\& Rahm M. Optics Express. E 18, 26 (2010)

11. Beruete M., Minin I. V., Minin O. V., Applied Physics Letter. 2014. E 105, 8 (2014) 